Psychotherapeut 2014 · 59:353-355

DOI 10.1007/s00278-014-1071-3

Online publiziert: 22. September 2014

(c) Springer-Verlag Berlin Heidelberg 2014

Renate Schepker ${ }^{1}$. Jörg M. Fegert ${ }^{2}$. Harald Freyberger ${ }^{3}$

${ }^{1}$ Abteilung Psychiatrie und Psychotherapie des Kindes- und Jugendalters, ZfP Südwürttemberg, Ravensburg

${ }^{2}$ Kinder und Jugendpsychiatrie, Universität Ulm

${ }^{3}$ Klinik und Poliklinik für Psychiatrie und Psychotherapie der Universitätsmedizin Greifswald, Helios Hanseklinikum Stralsund

\title{
Aufbruch zu einer patientenorientierten Psychotherapie des 21. Jahrhunderts
}

ten/Quartal und Therapeut in der Praxis mit durchschnittlich 3,5 Kontakten/Quartal und 2,3 Diagnosen/Quartal; Herpertz et al. 2011).

Eklatanter noch als für Erwachsene ist die Erreichbarkeit von Psychotherapie für Kinder und Jugendliche regional stark unterschiedlich - wenngleich es wünschenswert wäre, dass v. a. Jugendliche nicht auf elterliche Fahrdienste zur Therapie mit allen damit verbundenen Dynamiken angewiesen wären. Die Bertelsmann-Stiftung veröffentlichte anhand der Daten von 2011 einen gesamtdeutschen Überblick zur Erreichbarkeit von Psychotherapie durch Kinderund Jugendlichenpsychotherapeuten sowie Kinder- und Jugendpsychiater und konnte aufweisen, dass selbst bei Weglassen der 20 Kreise mit Extremwerten (gegenüber dem Bundesdurchschnitt von 29 betrug der Extremwert 150 Spezialisten/100.000 Einwohnern unter 18 Jahren) noch Schwankungen um Faktor 16,6 in Deutschland existieren. Mehr als 70\% aller Kreise lagen unter dem Bundesdurchschnitt. Von einer Versorgungsgerechtigkeit für Kinder und Jugendliche kann somit keine Rede sein, und die rar gesäten Institutsambulanzen können und sollen die Disparitäten in den großen Versorgungsgebieten (3-mal größer als für Erwachsene) nicht ausgleichen, da vielerorts die Pauschalen nicht ausreichend sind, um eine psychotherapeutische Behandlung lege artis durchzuführen.

Überdies ist, im Gegensatz zu einer doch auskömmlichen psychotherapeutischen Versorgung von Erwachsenen der Mittelschicht, d. h. Ersatzkassenversicher- ten (Gaebel et al. 2013), die Morbidität mit seelischen Störungen bei Kindern und Jugendlichen stark unterschichtlastig, und dies müsste entsprechend in einem fairen Zugang für alle Kinder gewährenden Versorgungssystem gewährleistet sein. Daten hierzu sind nicht bekannt - außer dass der KiGGS-Survey, folgerichtig im Duktus der bisherigen Ausführungen, feststellen musste, dass nur etwa die Hälfte aller als auffällig befundenen Kinder je einen Spezialisten aufgesucht hatte (Hölling et al. 2012). Die nächste Erhebungswelle des KiIGGS-Survey, die soeben erst veröffentlicht wurde, zeigt zwar insgesamt positive Trends im gesundheitlichen Befinden und Verhalten von Kindern und Jugendlichen in Deutschland, unterstreicht aber noch einmal sehr deutlich die Schichtabhängigkeit von Belastungen und Gesundheitsrisiken (Ziese 2014; Lange et al. 2014; Lampert et al. 2014; Ellert et al. 2014; Hölling et al. 2014; Schlack et al. 2014; Rattay et al. 2014).

Neben Schichtfaktoren können natürlich individuelle und familienbezogene Faktoren zu einer geringen Inanspruchnahme psychotherapeutischer Leistungen führen. Dies trifft sowohl zu, wenn betroffene Kinder Eltern mit psychischen Störungen und entsprechender Reserviertheit haben, wenn Eltern sich nicht auf eine gemeinsame Vorgehensweise einigen können oder wenn schlichtweg aus Gründen von Armut oder Sprachbarrieren bei Migrationshintergrund ein $\mathrm{Zu}$ gang nicht gefunden werden kann. All dies kann dazu beitragen, dass das Aufsuchen regelmäßiger ambulanter Behandlung nichtbewältigbar wird. Andererseits ter und werden niederfrequenter behandelt (laut KBV-Statistik 2010375 Patien- 
stellt eine stationäre Behandlung für Eltern eine ebenso große Belastung dar, ohne dass ein Transfer der erreichten Fortschritte in das häusliche Umfeld automatisch erfolgen würde. Die naheliegende Lösung der Schaffung aufsuchender Angebote wird in Deutschland kaum praktiziert. Demgegenüber wiesen Boege et al. (2014) die Praktikabilität für alle Diagnosen und sehr gute Erfolge im Sinne eines stationsersetzenden Angebots nach. Die Forschergruppe hat nun im vorliegenden Heft die Patienten- und Elternzufriedenheit mit diesem Angebot qualitativ und quantitativ erhoben sowie überragende Zufriedenheitsergebnisse erzielt. Besonders hervorzuheben ist, dass beiden Arbeiten ein randomisiertes kontrolliertes Design und ein längerer Katamnesezeitraum zugrunde liegen: Das hebt die Wissenschaftlichkeit der Ergebnisse auf ein hohes Niveau und belegt, dass mit der Flexibilisierung des Settings auch nachhaltige Erfolge erzielt werden können.

Eine besonders schwer erreichbare Gruppe ist die der Patienten mit Störungen des Sozialverhaltens. Diese Patientengruppe ist bei starker Störungsausprägung selten mit einem rein ambulanten Angebot zu halten, da viele Terminversäumnisse Teil des Störungsbilds sind, aber auch stets ein Herausfallen aus sozialen, schulischen und beruflichen Kontexten droht. In langfristiger ambulanter Richtlinienpsychotherapie findet sich diese Diagnosegruppe daher unterrepräsentiert. Unabhängig davon, welche Dynamik hinter der Genese dieser Störungen liegt, inszenieren die Jugendlichen im stationären Kontext oft disziplinarische Entlassungen. Das in diesem Heft vorgestellte Konzept in Form der Pilotstudie von Boege et al. hat hier mit dem Zugang einer gestuften Intervallbehandlung ermutigende erste Ergebnisse, insbesondere eine gute Haltefähigkeit sowie deutliche Verbesserungen des Verhaltens erreicht und hätte Replikationen verdient.

Die hervorzuhebende Besonderheit der vorgestellten Studien in diesem Band ist, dass insgesamt sehr flexibel mit der teuren Ressource stationärer Behandlung umgegangen wurde. Bei den Jugendlichen mit Störungen des Sozialverhaltens (Boege et al.) wurden durch den Intervallansatz die Mühen der kontinuierlichen Motiva- tionsfindung teilweise in den ambulanten Bereich hinausverlagert. Beim Vorgehen von Corpus et al. und Kirchmann et al. wurde analog zu den Teams in Praxen niedergelassener Kinder- und Jugendpsychiater mit SPV die sonst nur stationär mögliche Multiprofessionalität eines psychotherapeutischen Vorgehens in den außerstationären Raum, in diesem Fall noch weitergehend in den Lebensraum transportiert unter Verkürzung des Aufenthalts und Stärkung des Elterneinbezugs.

Nichtsdestotrotz gibt es Fallkonstellationen und Situationen, in denen eine langfristige stationäre Behandlung unausweichlich ist - ohne dass dadurch automatisch eine Chronifizierung der zugrunde liegenden Störung angezeigt wird oder ein dauerhafter Hospitalismus mit Reduzierung autonomer Fähigkeiten resultieren muss. Die Fallgeschichte einer Patientin mit der seltenen und bisher nicht im Spektrum der Internationalen statistischen Klassifikation der Krankheiten und verwandter Gesundheitsprobleme (ICD) vorkommenden Diagnose eines „pervasive refusal syndrome" kontrastiert und ergänzt die beiden anderen Arbeiten insofern, als es hier nicht um eine schnelle Entlassung gehen konnte, sondern im Gegenteil eine noninterventionistische Grundhaltung der "tender loving care“ angezeigt war.

Kinder und Jugendliche mit ausgeprägten Störungen brauchen - das mag der Gesamtduktus der Arbeiten in diesem Heft sein - äußerst flexible und individualisierte psychotherapeutische Settings in Zugang und Frequenz. Diese gehen über die klassische Zweipersonenpsychotherapie weit hinaus, erweitern und ergänzen das stationäre Setting und führten in den hier vorgelegten Studien zur aufsuchenden Behandlung sogar zu höherer Patienten- und Elternzufriedenheit als mit der vollstationären Behandlung. Nicht nur dass natürlicherweise Eltern aufgrund der bestehenden realen $\mathrm{Ab}$ hängigkeiten einbezogen werden müssen, sondern bei komplexer Problematik sind komplexere Lösungswege angezeigt.

\section{Korrespondenzadresse}

\section{Prof. Dr. Renate Schepker}

Abteilung Psychiatrie und Psychotherapie des Kindes- und Jugendalters, ZfP Südwürttemberg Weingartshofer Str. 2, 88214 Ravensburg renate.schepker@zfp-zentrum.de

\section{Einhaltung ethischer Richtlinien}

Interessenkonflikt. Renate Schepker, Jörg M. Fegert und Harald Freyberger geben an, dass kein Interessenkonflikt besteht.

\section{Literatur}

Bertelsmann-Stiftung (Hrsg) (o J) Psychische Versorgung von Kindern und Jugendlichen. http://www. faktencheck-gesundheit.de. Zugegriffen 31. Dezember 2011

Boege I, Corpus N, Schepker R (2014) Behandelt zu Hause Gesund werden. Z Kinder Jugendpsychiatr Psychother 42:27-37

Ellert U, Brettschneider A, Ravens-Sieberer U; KiGGS Study Group (2014) Gesundheitsbezogene Lebensqualität bei Kindern und Jugendlichen in Deutschland - Ergebnisse der KiGGS-Studie - Erste Folgebefragung (KiGGSWelle 1). Bundesgesundheitsblatt Gesundheitsforschung Gesundheitsschutz 57:798-806

Gaebel W, Kowitz S, Fritze J, Zielasek J (2013) Inanspruchnahme des Versorgungssystems bei psychischen Erkrankungen: Sekundärdaten von drei gesetzlichen Krankenkassen und der Deutschen Rentenversicherung Bund. Dtsch Arztebl Int 110(47):799-808. DOI 10.3238/arztebl.2013.0799

Herpertz S, Kruse J, Schaff C et al (2011) Die Zukunft der ärztlichen Psychotherapie. Expertise für die Bundesärztekammer anhand von Daten der KBV. http://www.bundesaerztekammer.de

Hölling H, Schlack R, Kamtsiuris P et al (2012) Die KiGGS-Studie. Bundesgesundheitsblatt Gesundheitsforschung Gesundheitsschutz 55:836-842

Hölling H, Schlack R, Petermann F, Ravens-Sieberer U, Mausz E; KiGGS Study Group (2014) Psychische Auffälligkeiten und psychosoziale Beeinträchtigungen bei Kindern und Jugendlichen im Alter von 3 bis 17 Jahren in Deutschland - Prävalenz und zeitliche Trends zu 2 Erhebungszeitpunkten (2003-2006 und 2009-2012). Ergebnisse der KiGGS-Studie - Erste Folgebefragung (KiGGS-Welle 1). Bundesgesundheitsblatt Gesundheitsforschung Gesundheitsschutz 57:807-819

Lampert T, Müters S, Stolzenberg H. Kroll LE; KiGGS Study Group (2014) Messung des sozioökonomischen Status in der KiGGS-Studie - Erste Folgebefragung (KiGGS-Welle 1). Bundesgesundheitsblatt Gesundheitsforschung Gesundheitsschutz 57:762-770

Lange M, Butschalowsky G, Jentsch F, Kuhnert R, Schaffrath Rosario A, Schlaud M Kamtsiuris P; KiGGS Study Group (2014) Die erste KiGGS-Folgebefragung (KiGGS Welle-1): Studiendurchführung, Stichprobendesign und Response. Bundesgesundheitsblatt Gesundheitsforschung Gesundheitsschutz 57: 747-761 
Rattay R, von der Lippe E, Lampert T; KiGGS Study Group (2014) Gesundheit von Kindern und Jugendlichen in Eineltern-, Stief- und Kernfamilien - Ergebnisse der KiGGS-Studie - Erste Folgebefragung (KiGGS-Welle 1). Bundesgesundheitsblatt Gesundheitsforschung Gesundheitsschutz 57:860-868

Schlack R, Mauz E, Hebebrand J, Hölling H; KiGGS Study Group (2014) Hat die Häufigkeit elternberichteter Diagnosen einer Aufmerksamkeitsdefizit-/Hyperaktivitätsstörung (ADHS) in Deutschland zwischen 2003-2006 und 2009-2012 zugenommen? Ergebnisse der KiGGS-Studie - Erste Folgebefragung (KiGGS-Welle 1). Bundesgesundheitsblatt Gesundheitsforschung Gesundheitsschutz 57:820-829

Ziese T (2014) KiGGS ist zurück: Welle 1. Bundesgesundheitsblatt Gesundheitsforschung Gesundheitsschutz 57:745-746

\section{Schwerpunktthemen}

Das Herausgebergremium der Zeitschrift Psychotherapeut lädt Autorinnen und Autoren ein, an den geplanten Schwerpunkten mitzuarbeiten und geeignete Manuskripte einzureichen. Diese werden dem üblichen Reviewverfahren unterzogen. Darüber hinaus freuen wir uns über die Zusendung freier (unaufgeforderter) Originalia zu selbstgewählten Themen.

Bitte schicken Sie Ihren Beitrag an die Redaktion:

Regine.Karcher-Reiners@springer.com

Die Schwerpunktplanung ist vorläufig und kann kurzfristig umdisponiert werden.

Heft 2/15: Zeit (LPW 2014)

(Deadline 30.10.2014)

Heft 3/15: Der psychotherapeutische Prozess

(Deadline 30.12.2014)

Heft 4/15: Selbstbeschädigung und Symptomatik autoaggressiven Verhaltens

(Deadline 30.02.2015)

Heft 5/15: Psychotherapie der Persönlichkeitsstörungen

(Deadline 30.04.2015)

Heft 6/15: Körperbild

(Deadline 30.06.2015) 